\title{
Management of lung nodules in Brazil-assessment of realities, beliefs and attitudes: a study by the Brazilian Society of Thoracic Surgery (SBCT), the Brazilian Thoracic Society (SBPT) and the Brazilian College of Radiology (CBR)
}

\author{
Maria Teresa Ruiz Tsukazan ${ }^{1,2}$, Ricardo Mingarini Terra ${ }^{3}$, Frank Detterbeck ${ }^{4}$, Ilka Lopes Santoro ${ }^{5}$, Bruno \\ Hochhegger $^{6,7}$, Gustavo de Souza Portes Meirelles ${ }^{8}$, Gustavo Fortunato ${ }^{9}$, Gustavo Faibischew Prado ${ }^{10,11}$ \\ ${ }^{1}$ Thoracic Surgery Division, Hospital São Lucas da Pontificia Universidade Catolica do Rio Grande do Sul (PUCRS), Porto Alegre, Brazil; \\ ${ }^{2}$ Pulmonary and Thoracic Surgery Division, Hospital Moinhos de Vento, Porto Alegre, Brazil; ${ }^{3}$ Thoracic Surgery Division, Instituto do Coracao, \\ Hospital das Clinicas HCFMUSP, Faculdade de Medicina, Universidade de Sao Paulo, Sao Paulo, Brazil; ${ }^{4}$ Thoracic Surgery, Yale University, \\ New Haven, USA; ${ }^{5}$ Respiratory Division, Escola Paulista de Medicina (EPM), Universidade Federal de São Paulo (UNIFESP), São Paulo, Brazil; \\ ${ }^{6}$ Radiology, Hospital São Lucas da Pontificia Universidade Catolica do Rio Grande do Sul (PUCRS), Escola de Medicina, Porto Alegre, Brazil; \\ ${ }^{7}$ Radiology, Universidade Federal de Ciências da Saúde de Porto Alegre (UFCSPA), Porto Alegre, Brazil; ${ }^{8}$ Radiology, Grupo Fleury, São Paulo, \\ Brazil; 'Thoracic Surgery Division, Santa Casa de Misericórdia da Bahia, Salvador, Brazil; ${ }^{10}$ Pulmonary Division, Instituto do Coracao, Hospital das \\ Clinicas HCFMUSP, Faculdade de Medicina, Universidade de Sao Paulo, Sao Paulo, Brazil; ${ }^{11}$ Pulmonary Division, Instituto do Cancer do Estado de \\ Sao Paulo "Octavio Frias de Oliveira", Faculdade de Medicina, Universidade de Sao Paulo, Sao Paulo, Brazil \\ Contributions: (I) Conception and design: All authors; (II) Administrative support: RM Terra; (III) Provision of study materials or patients: All authors; \\ (IV) Collection and assembly of data: RM Terra; (V) Data analysis and interpretation: MTR Tsukazan, RM Terra, F Detterbeck; (VI) Manuscript \\ writing: All authors; (VII) Final approval of manuscript: All authors. \\ Correspondence to: Ricardo Mingarini Terra. Avenida Dr. Enéas de Carvalho Aguiar, 44, Bloco II, Sala 9, Cerqueira César, CEP 05403-000, São \\ Paulo, SP, Brasil. Email: rmterra@uol.com.br.
}

Background: Pulmonary nodules are common; some are inconsequential while others are malignant. Management of solitary pulmonary nodule (SPN) in Brazil appears to be highly variable, potentially leading to suboptimal outcomes. Assessment of the variability and the association with the degree of availability of resources can provide a foundation for development of clinical guidelines for management of SPN specific for the Brazilian setting.

Methods: A web-based survey was developed by thoracic surgeons, pulmonologists and radiologists to evaluate SPN perception and management. This survey was sent to their respective national societies members and answers collected between August and December 2016. That included multiple choice questions regarding age, specialty, SPN management, accessibility to exams and interventional procedures characterizing public (SUS) and supplementary private working settings.

Results: A total of 461 questionnaires were answered. More than half of participants live in cities with over one million people. Specialties were reasonable equilibrated with $43.5 \%$ radiologists, $33.5 \%$ thoracic surgeons, $20.3 \%$ pulmonologists and $2.6 \%$ others. Most of the respondents work in both public and private sector $(72.7 \%)$. Private has a similar reality compared to well-developed nations regarding exams accessibility and interventions. SUS setting has a significant variability access according to the participants. CT is only easily available in $31.9 \%$ of cases, PET-CT is easily available in $24.4 \%$, bronchoscopy is easily available for $42.8 \%$, transthoracic needle biopsy is only easily available in $13.9 \%$ and video-assisted thoracoscopic surgery (VATS) biopsy is not available in $19.5 \%$. When there is a probability of malignancy of $50 \%$ or higher, $46.5 \%$ of participants would be comfortable recommending surgical biopsy. When the probability is higher than $10 \%$, only $36.9 \%$ would be comfortable following up radiologically.

Conclusions: Brazil has a very different setting for public and private patients regarding exams accessibility and management options. That might explain why participants have a higher tendency to choose 
interventional diagnosis and explains why current guidelines may not be applicable to developing countries reality.

Keywords: Lung neoplasm; guideline; mass screening; surveys and questionnaires; lung; lung cancer

Submitted Dec 18, 2017. Accepted for publication Apr 03, 2018.

doi: $10.21037 /$ jtd.2018.05.23

View this article at: http://dx.doi.org/10.21037/jtd.2018.05.23

\section{Introduction}

The nature of a solitary pulmonary nodule (SPN) varies from inconsequential to potentially lethal. Sorting out it is can be challenging. A scientifically based structured approach can reduce variability, minimize unnecessary interventions and optimize treatment when it is needed; major guidelines have been developed to accomplish this, including the American College of Chest Physicians (ACCP) (1), British Thoracic Society (BTS) (2), National Comprehensive Cancer Network (NCCN) (3) and others (4). However, it appears that these guidelines have had limited impact in Brazil, and variability and uncertainty in the management of SPN is frequent in Brazil.

It is unclear to what degree international guidelines are applicable in Brazil that belongs to the 30 tuberculosis (TB) high-burden countries responsible for $87 \%$ of worldwide TB cases (5). This complicates evaluation of a SPN (6). Furthermore, Brazil is a large country with very heterogeneous regions and healthcare settings. The healthcare system is composed of a universal accessible Public Healthcare System (SUS) and a Private Healthcare System (Supplementary Healthcare System), which covers some employees and wealthier people. There is variability in the availability of resources (CT, PET-CT etc.) and interventions [bronchoscopy, video-assisted thoracoscopic surgery (VATS) etc.] across the country in both the public and supplementary Healthcare System.

This project was initiated to develop an assessment of how SPN are managed in Brazil and the availability of management options. We wanted to know the perspectives across different specialties and how appropriate existing SPN guidelines can be in this setting. This would provide a basis for development of a thoughtful structure to guide management of SPN specifically tailored to Brazil.

\section{Methods}

A project to develop a Brazil-specific guideline for SPN was initiated in 2015. This is collaborative undertaking of the Brazilian Society of Thoracic Surgery (SBCT), the Brazilian Thoracic Society (SBPT), the Brazilian College of Radiology (CBR), the Brazilian Society of Medical Oncology (SBOC) as well as the American College of Chest Physicians (ACCP). The early phase of this undertaking led to the recognition of the need for a better understanding of the current situation in Brazil with respect to SPN.

The Brazilian Nodule Guideline group developed a survey (find in the Appendix the original instrument in Portuguese and a version translated into English) which was approved by University of Sao Paulo Ethics Committee by the ID CAAE:59712616.7.0000.0065. A draft version was piloted within a select subgroup of SBCT (general thoracic surgeons), SBPT (all pulmonologists) and CBR (all radiologists) members. The refined web-based survey addressed questions of demographic data, threshold to use invasive methods, availability of follow up and invasive methods in public and private settings; skip logic was used to direct the responders to relevant questions and skip over those not germane to the individual.

Between August 2016 and December 2016, the survey was sent to the members of the SBCT, SBPT and CBR with 2 rounds of reminders. Participants were informed their participation was voluntary and anonymous. Study data were collected and managed using REDCap electronic data capture tools hosted at University of Sao Paulo, Brazil (7). REDCap (Research Electronic Data Capture) is a secure, web-based application designed to support data capture for research studies, providing (I) an intuitive interface for validated data entry; (II) audit trails for tracking data manipulation and export procedures; (III) automated export procedures for seamless data downloads to common statistical packages; and (IV) procedures for importing data from external sources.

Relative distributions were estimated according to the number of respondents to each question. Missing data were ignored, and no value attributed. Fisher exact test 
Access to healthcare resources in the private and public systems

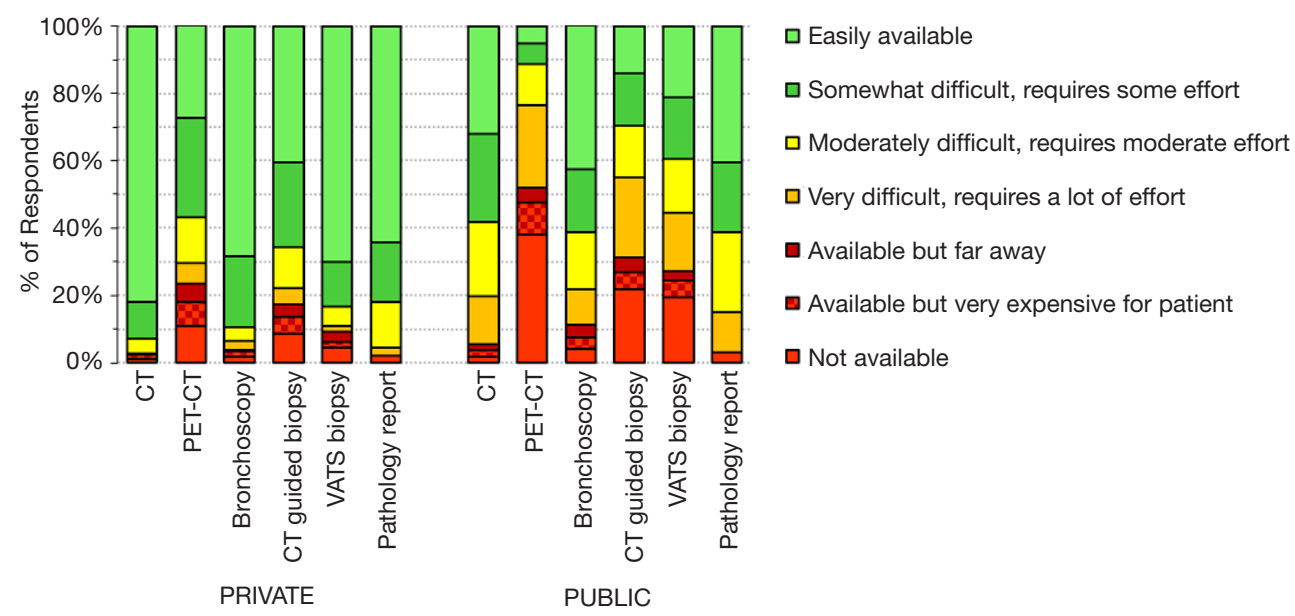

Figure 1 Degree of access to exams according to patient insurance (public or private). Scale for pathology report confidence: not available (red), difficult, sometimes requires $2^{\text {nd }}$ opinion (orange), moderately reliable (yellow), usually reliable (green), available and reliable (light green).

analysis was used for comparisons. A probability value of $<0.05$ was considered statistic significant. We divided city size into three categories based on population: $<500,000$, $500,000-1,000,000$ and $>1$ million to evaluate differences in public health exams and procedures access. To explore how the availability of tests and procedures might influence the management responses, we categorized the access to particular tests and procedures as either good, intermediate or poor. Because the majority of physicians work in both sectors, and because the thresholds at which physicians were comfortable with particular management strategies was general and not sector-specific, these accessibility categories represent a combination of private and public systems. Good access is defined as a test or procedure being either easily available, somewhat difficult and requires some effort or moderately difficult and requires moderate effort for both healthcare systems. Intermediate access was assigned when access in one healthcare systems was good and the other was either very difficult and requires a lot of effort, available but far away, available but very expensive for patient, or not available. Finally, when access in both systems was very difficult and requires a lot of effort, available but far away, available but very expensive for patient, or not available, the access was considered poor.

\section{Results}

A total of 461 answers were received; the response rate was $27 \%$ for SBCT, $5 \%$ for SBPT and $2 \%$ for CBR (the
CBR consists of all radiologists with only a small minority that are thoracic radiologists). Pulmonologists account for $20 \%$ [94] of respondents, radiologists $44 \%$ [201], thoracic surgeons 33\% [155] and others 3\% [12]. There was a broad age distribution; $34 \%$ were $<35$ years old, $38 \% 35-50$ years old, 24\% 51-65 years old and only $6 \%>65$ years old. Most of the respondents work in both the public and private sectors $(73 \%)$, only $7 \%$ of respondents work exclusively in public system and $20 \%$ exclusively in private system. More than half of participants (59\%) work in a city with one million or more population followed by intermediate city size between 100,000 and 500,000 population (23.4\%). Number of SPN new cases seen per month varies from 6 to $20(39 \%), 2$ to $5(29.5 \%)$ and more than $20(24.5 \%)$.

There was marked variability in the availability of imaging and procedures (Figure 1). CT is not universally available in the public system. PET-CT is usually very difficult to access in the public system (unavailable for $38 \%$ of the public health population). Bronchoscopy is easily available in the private (supplementary) system for $68 \%$ of respondents $v s .43 \%$ in the public healthcare system. CTguided biopsy is easily available to only $14 \%$ of respondents in the public system $v$ s. to $40 \%$ in the private system. A VATS surgical biopsy for diagnosis of a SPN is easily accessible to $21 \%$ of respondents in the public system $v$ s. to $70 \%$ in the private system $(\mathrm{P}<0.001)$.

There were marked differences in the availability of tests in the public system by city size, particularly for more expensive and sophisticated exams. In smaller cities 


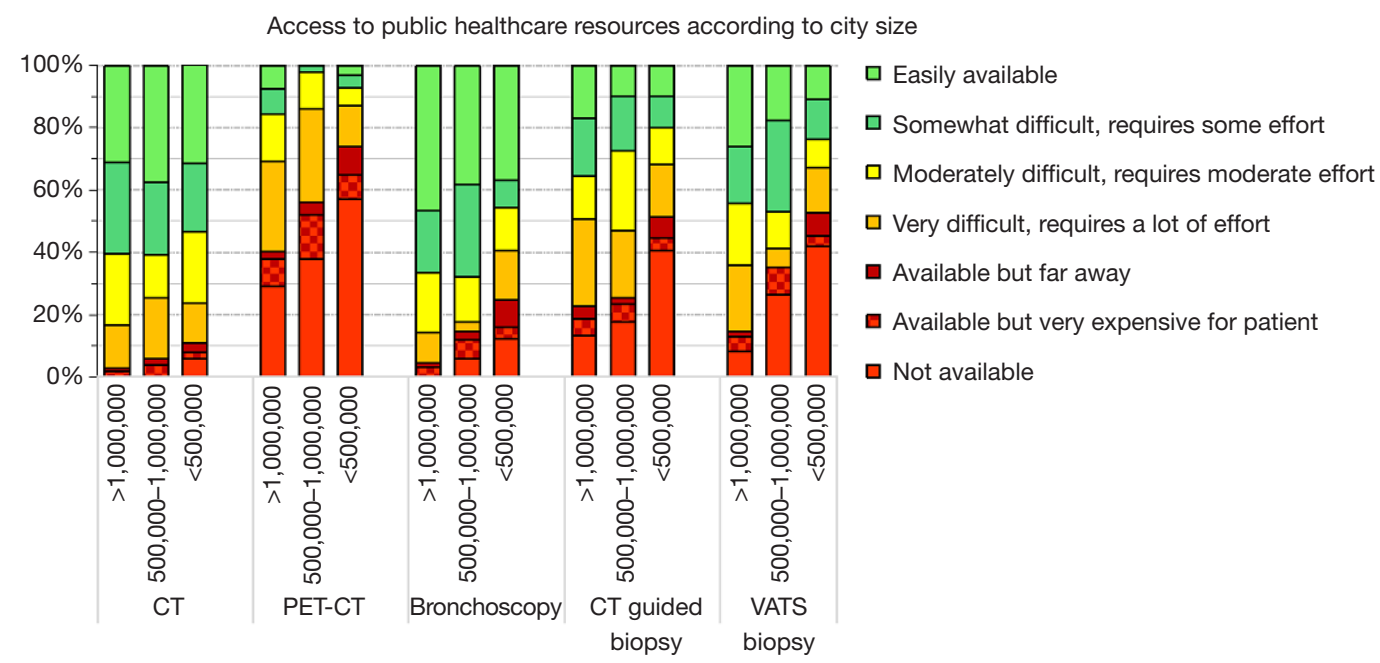

Figure 2 Degree of access to various exams in the public healthcare system by city size (population) and are all statistically significant $(\mathrm{P}<0.05)$.

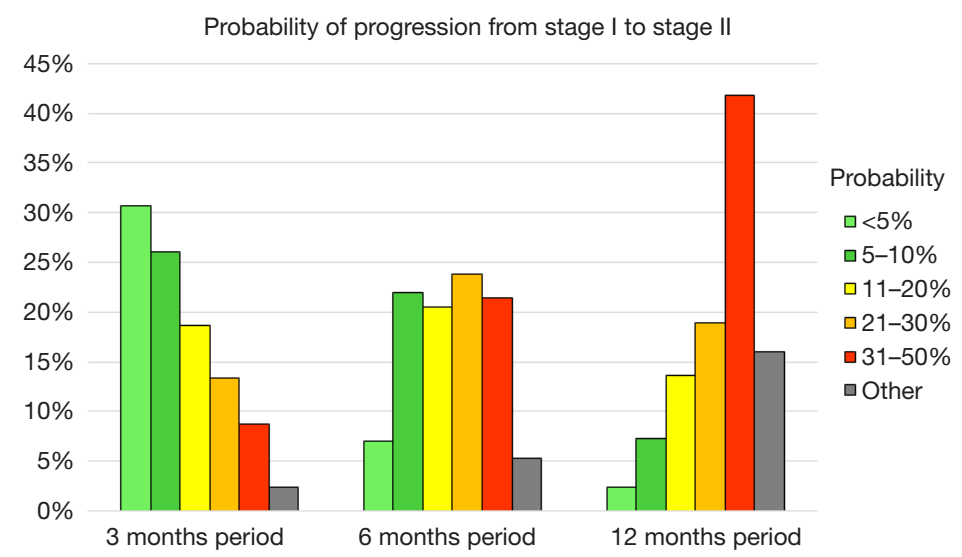

Figure 3 Perception of chance of stage progression during a period of observation by specialist (pulmonologist, radiologist and thoracic surgeon). Differences across specialties are not are statistically significant $(\mathrm{P}>0.05)$.

PET-CT was not available in 57\%, bronchoscopy was not available for $12 \%$, CT guided biopsy in $41 \%$ and VATS biopsy was not available in $42 \%$ (Figure 2). In contrast, in cities $>1,000,000$ PET-CT was not available in $29 \%$, bronchoscopy is accessible to everyone (although sometimes with some difficulty), CT guided biopsy was not available in $13 \%$ and VATS biopsy not available in $8 \%$. All differences were statistically significant $(\mathrm{P}<0.05)$.

About half of respondents believed there was a substantial rate of progression from stage I to stage II within 3 months, only $1 / 3$ thought the risk was $<10 \%$ within 6 months (Figure 3). There was little difference in this assessment according to the specialty of the respondent.

Regarding guidelines familiarity, only a minority (20\%) reported that they were unfamiliar with SPN guidelines, and most reported using them: $46 \%$ use it often, $31 \%$ used a couple times, and $22 \%$ never used it. The respondents are more familiar with ACCP guidelines (43\%), followed by NCCN (16\%) and BTS (8\%) guidelines for SPN.

We explored physician's attitudes regarding aggressiveness of management of SPN by asking whether they would be comfortable, given a particular estimate of the probability that a SPN is a lung cancer, with observation (serial imaging) or with proceeding to a surgical biopsy. Only $37 \%$ would be comfortable following a patient for any period of time when the probability of cancer is estimated to be $10 \%$; less than $10 \%$ would feel comfortable when the probability of cancer is estimated to be $50 \%$ (Figure 4). There were no 


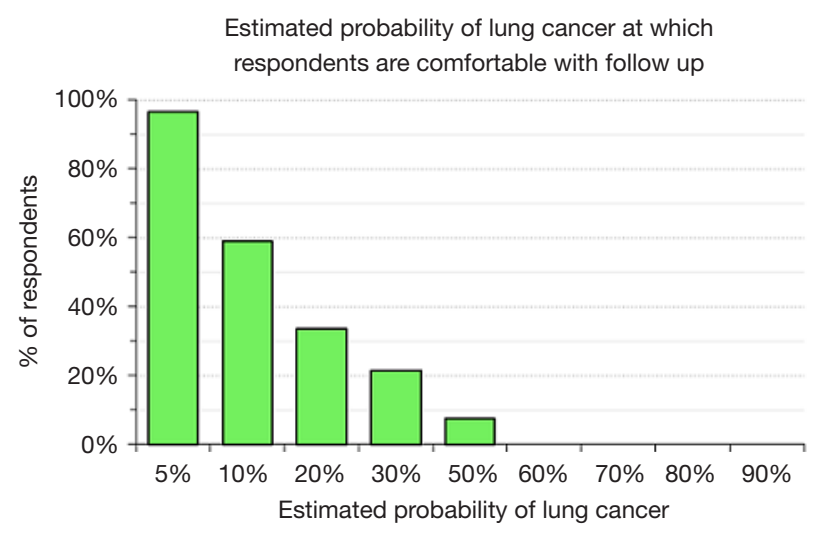

Figure 4 Estimated probability that a SPN is a lung cancer at which respondents were comfortable with an observation strategy (serial imaging). (The option "other" was chosen by $3.5 \%$ ). SPN, solitary pulmonary nodule.

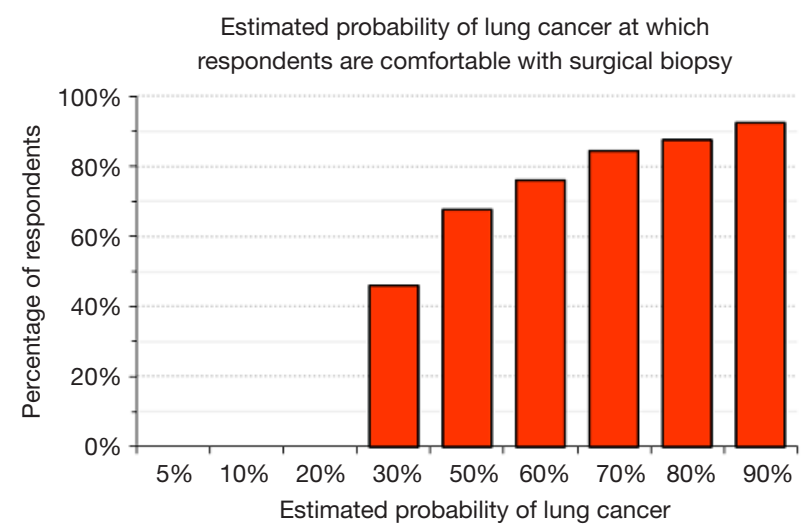

Figure 5 Estimated probability that a SPN is a lung cancer at which the respondents were comfortable proceeding with surgical biopsy. (Options $<30 \%$ were not offered in the questionnaire. The option "other" was chosen by $7.6 \%$ ). SPN, solitary pulmonary nodule.

significant differences among categories of city size $(\mathrm{P}>0.72)$ and medical specialties $(\mathrm{P}>0.38)$. The probability of lung cancer threshold at which respondents felt comfortable sending a patient to a surgical biopsy was relatively low: $46 \%$ felt comfortable recommending surgical biopsy when the probability of lung cancer was $30 \%$ and $68 \%$ were comfortable when the probability was $50 \%$ (Figure 5). These answers were not significantly different among categories of city size $(\mathrm{P}>0.35)$ or specialty $(\mathrm{P}>0.37)$.

Using the categorized access of tests and procedures we found the accessibility did not affect the aggressiveness of
SPN management. Access to PET-CT was most variable across categories of city size and type of healthcare system; we found that physicians with good access to PET-CT tended to be more conservative recommending surgical biopsy and physicians with poorer infrastructure were more aggressive (Figure 6); however, the difference was not significant $(\mathrm{P}=0.21)$. The only exam that significantly $(\mathrm{P}<0.002)$ affected the readiness to recommend surgical biopsy was the access to CT guided biopsy. The probability of lung cancer in a SPN at which respondents were comfortable with serial imaging was not affected by the availability of any exam or procedure (CT, PET-CT, bronchoscopy, CT guided biopsy, VATS biopsy).

We also evaluated if the degree of familiarity or usage of guidelines correlated with respondents' attitudes regarding surgical lung biopsy. Surprisingly, no differences were noted (Figure 7).

\section{Discussion}

This survey confirms that there is significant variability in resource availability with respect to tests and inventions relevant to management of SPN. Like many countries in Latin America, Brazil has both a public and private healthcare system. The availability of exams differs between these systems, particularly for sophisticated exams as PET-CT or less invasive biopsy options as transthoracic CT guided or VATS. Furthermore, the availability of exams is more limited outside of the larger cities for both healthcare systems.

The ACCP Evidence based guideline, which is most familiar to our participants, recommends surgical biopsy when the probability of lung cancer is high ( $>65 \%)(1)$. For SPN with a low probability of malignancy $(\leq 5 \%)$ observation with serial CT is recommended. When the probability is moderate (5-65\%), ACCP recommends a PET-CT (if the lesion is solid and $>8 \mathrm{~mm}$ ). If PET$\mathrm{CT}$ is negative, observation is generally used, although non-surgical biopsy can be considered if the probability of malignancy remains intermediate (10-65\%). If PETCT demonstrates moderate or intense uptake, a biopsy is recommended (either non-surgical or surgical). The NCCN and British guidelines similarly rely heavily on PET-CT in the management algorithm (2,3).

Brazilian physicians do not appear to follow the existing guidelines. Although $80 \%$ of participants affirmed they use guidelines, surprisingly $76 \%$ of Brazilian physicians were comfortable proceeding to surgical biopsy with a probability of $<60 \%$. However, the variable availability of PET-CT 


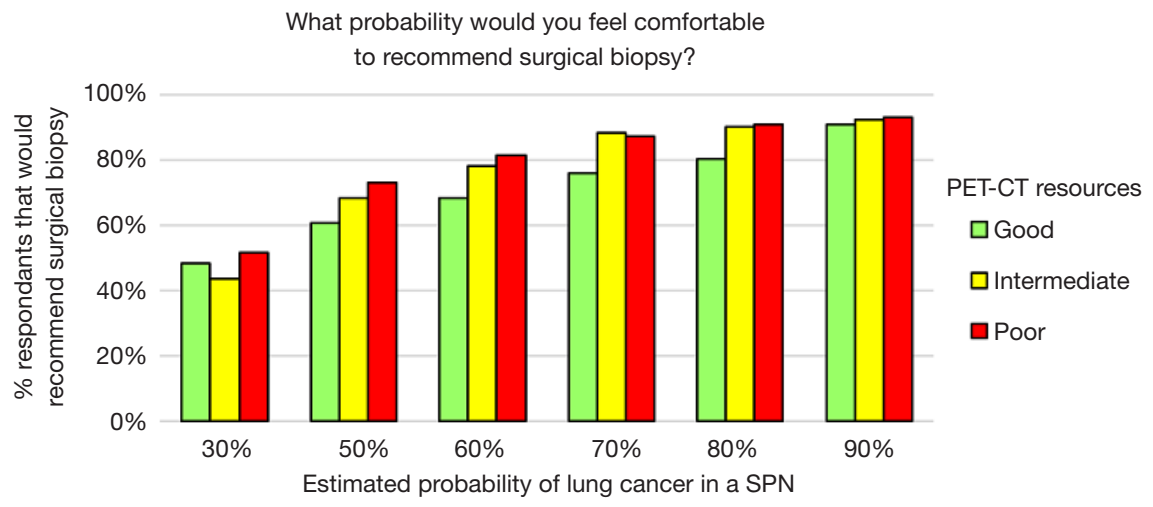

Figure 6 Threshold of the probability of lung cancer in a SPN at which respondents felt comfortable recommending a surgical biopsy according to the access to PET-CT. The difference was significant among PET-CT resources $(\mathrm{P}<0.002)$. See legend for Figure 6 for definition of good, intermediate and poor. (Options $<30 \%$ were not offered in the questionnaire. The option "other" was chosen by $9.1 \%$, $7.6 \%$ and $7.1 \%$ of respondents with good, intermediate or poor access to PET-CT, respectively). SPN, solitary pulmonary nodule.

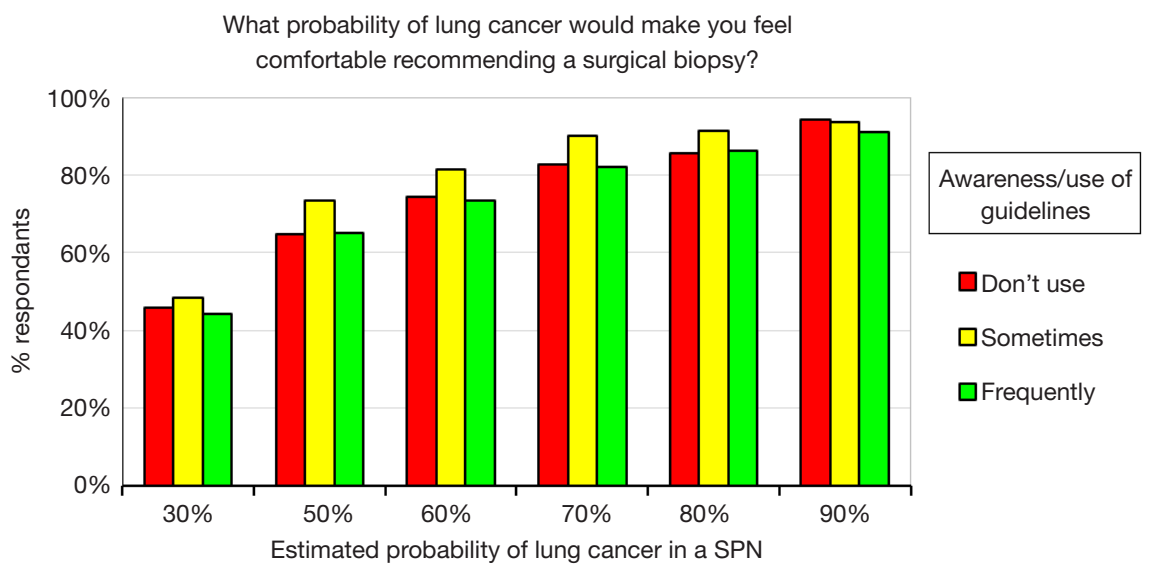

Figure 7 Threshold of the probability of lung cancer in a SPN at which respondents felt comfortable recommending a surgical biopsy according to the respondent's use of clinical guidelines. Differences are not significant $(\mathrm{P}=0.54)$. SPN, solitary pulmonary nodule.

suggests that these external guidelines may not be applicable to Brazil. In fact, our limited exploration suggests that at least for some exams, the availability significantly affected the management decisions. Furthermore, existing guidelines from the US and UK do not take into account the higher incidence of TB in Brazil. These observations suggest that simple translation and adoption of existing guidelines in Brazil is not a useful approach.

A Brazil-specific guideline must reflect evidence-based medicine but use this to make recommendations that are feasible in the Brazilian setting. In addition, because of the variability within Brazil, the structure of a Brazilianspecific guideline must be adaptable enough to apply to the variety of settings in Brazil. Finally, because the settings are generally more nuanced than simply available or not available (e.g., difficult, requires a lot of effort, available but far away), an assessment of the trade-offs involved is needed. This enhances the ability to make a rational decision about whether it is worth the effort to get a test or procedure with limited availability, or to focus on expediency and feasibility by choosing an alternative approach.

There are few similar studies to which our study can be compared. We are unaware of a similar survey done in another country. A study in Singapore, consisting of a retrospective review of 60 patients with lung cancer that presented as a SPN raised similar concerns: the high rate of TB led to frequent delay in diagnosis, questionable applicability of probability of lung cancer calculators, few 
patients managed according to ACCP guidelines (including specifically a paucity of PET-CT) and variability in management (6). A US-based survey of SPN management found moderate deviation from US-based management guidelines (8). There was less deviation $(20 \%)$ when there was low probability of lung cancer, and more with an intermediate probability or high probability of lung cancer. Notably, deviation from guideline standards were seen more commonly with those with less specialty training or less clinical experience (8).

The survey respondents harbored significant concerns about progression of early stage lung cancer. Approximately half of respondents thought there was at least a $10 \%$ chance of progression from stage I to stage II within 3 months, at least a $20 \%$ chance in 6 months and at least a $30 \%$ chance in 12 months. This rate has not been well defined, but existing data suggests it is likely much lower. A recent large database study of lung cancer found that a 2.5 -month delay was associated with a $1.7 \%$ higher rate of upstaging to $\mathrm{pII}$ or greater compared with early surgery (18.3\% vs. 16.6\%) (9).

This study has limitations. The survey was sent to all members of the relevant specialty societies in Brazil and the response rate is comparable to what is usually achieved in an email survey (8). It's very important to highlight that Brazil has a unique scenario regarding the SPN referral and management. It's more often to a general practitioner, cardiologist or oncologist find a SPN than a pulmonologist. In addition, those patients are referred straight to the thoracic surgeon, bypassing the pulmonologist. Therefore, even with a low response rate from pulmonologist, we believe the responses of the survey represent the majority of physicians managing SPN in Brazil. Although we did not ensure that subgroups (regions, age cohorts etc.) were sufficiently represented, we believe that it provides a reasonable cross section of Brazilian physicians. The survey was designed as a compromise between being short enough to encourage broad participation and being detailed enough to fully understand the reason behind observations. For example, regarding thresholds for comfort with a particular management approach we did not ask separately whether the respondent was answering for patients in the public or private system (or both). We are not able to definitively answer whether availability of resources affects management attitudes. Finally, the responses are subjective and qualitative. However, the survey was designed as a preliminary assessment of variability and potential associations. The survey provides direction in how to structure a guideline in Brazil for management of
SPN, even if it does not fully elucidate the reasons behind physicians' current management strategies.

\section{Conclusions}

There is variability in the access of tests and procedures to manage SPN in Brazil, reflecting at least in part differences in the healthcare systems (public and private) and location (city size). There is also variability in attitudes toward how to manage SPN. The existing beliefs do not mirror the available evidence and management strategies do not match available US or UK guidelines. However, the etiology of SPN in Brazil may be different than in other parts of the world, and the access to some tests and procedures limits the applicability of external guidelines. This underscores the need to thoughtfully assess the evidence pertaining to SPN in Brazil, and to develop a Brazil-specific guideline to help physicians manage these patients in an evidence-based yet realistic and feasible manner that is applicable to their particular setting.

\section{Acknowledgements}

The authors would like to thank Brazilian Society of Thoracic Surgery (SBCT), Brazilian Thoracic Society (SBPT), Brazilian College of Radiology (CBR) and Leticia Leone Lauricella.

\section{Footnote}

Conflicts of Interest: The authors have no conflicts of interest to declare.

Ethical Statement: The study was approved by University of Sao Paulo Ethics Committee by the ID CAAE:59712616.7.0000.0065.

\section{References}

1. Gould MK, Donington J, Lynch WR, et al. Evaluation of individuals with pulmonary nodules: when is it lung cancer? Diagnosis and management of lung cancer, 3rd ed: American College of Chest Physicians evidence-based clinical practice guidelines. Chest 2013;143:e93S-e120S.

2. Baldwin DR, Callister ME; Guideline Development Group. The British Thoracic Society guidelines on the investigation and management of pulmonary nodules. Thorax 2015;70:794-8.

3. NCCN Non-Small Lung Cancer Guidelines. Available 
online: https://www.nccn.org/professionals/physician_gls/ default.aspx\#nscl

4. MacMahon H, Austin JH, Gamsu G, et al. Guidelines for management of small pulmonary nodules detected on CT scans: a statement from the Fleischner Society. Radiology 2005;237:395-400.

5. World Health Organization. Tuberculosis. Available online: http://www.who.int/tb/publications/global_report/en/

6. Phua CK, Sim WY, Sen Tee K, et al. Evaluation of pulmonary nodules in Asian population. J Thorac Dis 2016;8:950-7.

7. Harris PA, Taylor R, Thielke R, et al. Research electronic

Cite this article as: Tsukazan MT, Terra RM, Detterbeck F, Santoro IL, Hochhegger B, Meirelles GS, Fortunato G, Prado GF. Management of lung nodules in Brazil-assessment of realities, beliefs and attitudes: a study by the Brazilian Society of Thoracic Surgery (SBCT), the Brazilian Thoracic Society (SBPT) and the Brazilian College of Radiology (CBR). J Thorac Dis 2018;10(5):2849-2856. doi: 10.21037/jtd.2018.05.23 data capture (REDCap)--a metadata-driven methodology and workflow process for providing translational research informatics support. J Biomed Inform 2009;42:377-81.

8. Iaccarino JM, Simmons J, Gould MK, et al. Clinical Equipoise and Shared Decision-making in Pulmonary Nodule Management. A Survey of American Thoracic Society Clinicians. Ann Am Thorac Soc 2017;14:968-75.

9. Samson P, Patel A, Garrett T, et al. Effects of Delayed Surgical Resection on Short-Term and Long-Term Outcomes in Clinical Stage I Non-Small Cell Lung Cancer. Ann Thorac Surg 2015;99:1906-12; discussion 1913. 